\title{
Impact of cusp inclinations on dental fractures in cracked tooth syndrome model and relevant risk evaluation
}

\author{
NINA XIE ${ }^{1,2}$, PENGLAI WANG ${ }^{1}$, CUI WU ${ }^{1}$, WENTING SONG ${ }^{2}$, WEN WANG $^{1}$ and ZONGXIANG LIU ${ }^{1}$ \\ ${ }^{1}$ Department of Peridontal Mucosa, The Affiliated Xuzhou Stomatology Hospital of Xuzhou Medical University, \\ Xuzhou, Jiangsu 221006; ${ }^{2}$ Department of Endodontics, School of Stomatology, \\ Xuzhou Medical University, Xuzhou, Jiangsu 221004, P.R. China
}

Received May 30, 2017; Accepted September 27, 2017

DOI: $10.3892 /$ etm.2017.5285

\begin{abstract}
We explored the impact of cusp inclinations on dental fractures in cracked tooth syndrome model and formulated corresponding risk scale. Forty maxillary premolars were randomized into four groups for cusp inclination measurements by digital radiovisiography (RVG). For cracked tooth models, buccal and palatal cusp inclinations were achieved by grinding in groups I $\left(59^{\circ}-50^{\circ}\right)$, II $\left(64^{\circ}-55^{\circ}\right)$ and III $\left(69^{\circ}-60^{\circ}\right)$, with group IV as blank control. All groups underwent compression loading test, with fracture levels recorded for statistical analysis. The fracture modes included a majority of crown root fractures and a minority of crown fractures in groups I and II, exclusive crown root fractures in group III, and exclusive crown fractures in group IV. Overall, palatal fractures were predominant versus buccal fractures, with exclusive palatal fractures in group IV, and oblique fractures were overwhelming versus the scanty vertical fractures. Fracture risk classification: grade III was prevalent in groups I and II, grade IV in group III, and grades I and II in group IV only. The fracture risk scores in groups III and IV had significant statistical differences versus groups I and II $(\mathrm{P}<0.05)$, with insignificant differences between groups I and II, respectively ( $P>0.05)$. Cracked teeth are more vulnerable to complex fractures, with increment of cusp inclinations contributable to complex fracture modes, involving deep roots and high risk scores.
\end{abstract}

\section{Introduction}

Teeth are crucial to the survival of most mammals $(1,2)$. The basic tooth structure consists of a brittle and stiff enamel shell,

Correspondence to: Dr Zongxiang Liu, Department of Peridontal Mucosa, The Affiliated Xuzhou Stomatology Hospital of Xuzhou Medical University, 130 West Huaihai Road, Xuzhou, Jiangsu 221006, P.R. China

E-mail:xzkqyylzx@163.com

Key words: cracked tooth syndrome, cusp inclination, fracture mode, fracture risk score encasing a tough and compliant dentin interior $(3,4)$. Despite their durability, tooth fractures are daily encountered by dentists (5). As known, tooth cracks have become the third largest cause of tooth loss after dental caries and periodontal disease (6). Tooth cracks could lead to all kinds of symptoms, and patients always fail to receive treatment timely when the tooth has no obvious symptom. Most patients with cracks often see a dentist after suffering pulpitis and periapical periodontitis, or even severe fracture (7). A variety of different clinical symptoms of tooth cracks depend on the depth of crack, with the increase of crack depth, clinical symptoms may be obvious and aggravating (8). The symptomatology described by the patients included localized pain during chewing or biting, inexplicable sensitivity to cold, as well as pain on release of pressure (9-12). Additionally, there are no measures to interfere with crack development.

Tooth cracks have a multi-factorial etiology, in which several dominant categories are classified, i.e. tooth anatomical morphology, biomechanical factors, iatrogenic causes and miscellaneous factors $(9,13)$. It is also well established that the high and steep cusp inclinations of tooth plays a vital role in tooth cracks (9). Related studies indicate that the horizontal component of occlusal force at the bottom of the fovea changes along with the cusp inclinations. The value of horizontal component will increase with the increase of cusp inclinations (14). Steep cusp inclines and deep grooves have been of concern as one of the predisposing factors for the incidence of tooth fracture in posterior natural dentition (15). Therefore, the cusp inclinations has a significant influence on tooth fractures. This indicates an effective reduction of cuspal inclination to the compromised teeth for dentists (16).

There are many methods for the treatment of cracked teeth, according to the sites and extent of the fracture $(17,18)$, i.e. immediate, direct restorations placed intracoronally without cuspal coverage, direct restorations, which provide cuspal coverage, indirect restorations placed intracoronally without any cuspal support and indirect restorations which provide cuspal coverage (onlays and full coverage restorations). While there is no universally accepted restorative treatments, it is generally agreed to keep fixed part of the tooth for moving on loading. The latter may be achieved in a limited number of cases simply by the removal of the affected cusp and restoring the defect with an appropriate material, or in the majority of 
cases by the placement of a restoration that prevents independent movement of the tooth segments on either side of the crack (11). Therefore, early evaluation of the extent of cracks has important role in therapy and prognosis.

Herein, we aimed to investigate the impact of cusp inclination on dental fracture by means of stress analysis by creating a simulated artificial tooth-crack model, with the degrees of risks estimated and risk scale formulated, this may provide certain theoretical bases for treatment plans of tooth cracks, and the evaluation of long-term prognosis.

\section{Materials and methods}

Specimen preparation. This study was approved by the Ethics Committee of the Affiliated Xuzhou Stomatology Hospital of Xuzhou Medical University. All enrolled outpatients undergoing orthodontic extraction provided written informed consent and a total of 70 maxillary premolars with intact radices dentis were included. Exclusion criteria: abnormal morphology; dental caries, severe wear or erosion, crack and defect; filling treatment; root canal therapy; root no. $\geq 2$; curved root canal $>30^{\circ}$.

Measurements of cusp inclination. The cusp inclination of extracted maxillary premolars was measured by digital radiovisiography (RVG). The mean buccal cusp inclination was $49 \pm 1.6^{\circ}\left(95 \% \mathrm{CI}, 48.6^{\circ}-49.3^{\circ}\right)$ and palatal cusp inclination was $40 \pm 1.9^{\circ}\left(95 \% \mathrm{CI}, 39.7^{\circ}-40.3^{\circ}\right)$. To obviate bias, 40 maxillary premolars with the cusp inclination of $95 \%$ CI were enrolled. All eligible teeth underwent cleansing of soft tissues and calculi, and were stored at room temperature in normal saline prior to experiment.

Establishment of cracked tooth model. Forty maxillary premolars were randomized into four groups $(n=10)$. The bias angle of mean mediobuccal and mediolingual cusp inclination in CTS was reportedly $9.29^{\circ}$ and $9.02^{\circ}$ (16), respectively, in order to create a simulated artificial tooth-crack model, as compared with the value of the reported cusp inclinations, thereby maxillary premolars in groups I, II and III as CTS groups were fabricated by increment of cusp inclination by $10^{\circ}, 15^{\circ}$ and $20^{\circ}$, with those intact teeth in group IV as controls (Table I).

RVG was employed to measure the height (the distance from cusps to the central fossa plane) and width (the distance from cusps to the dental longitudinal axis plane) of the buccal and palatal tooth cusps in the experimental groups for planar mapping (Fig. 1). To simulate the de facto cusp inclinations in CTS, parameters were adjusted with the aid of vernier caliper (precision at $0.02 \mu \mathrm{m}$ ) and diamond burs for pre-crack on the $2 / 3$ of mesiodistal diameter of the crown and the height to the cementoenamel junction (CEJ) by the \#700 drill. The severe and irregular abrasion of the tooth will make the cusps steeper in clinical practice, thus generating the wedging effect which could lead to the tooth cracks and fractures. It is required to maintain the integrity of the bottom of the fovea as far as possible for simulating clinical condition of such steep cusps during the adjustment of cuspal inclination. The grinding of cusps is confined to the enamel layer without involving the dentin. Therefore, the partial enamel on the cuspal slopes are
Table I. Grouping of tooth tip samples.

\begin{tabular}{lll}
\hline Group & $\mathrm{N}$ & \multicolumn{1}{c}{ Group descriptions } \\
\hline 1 & 10 & Buccal cusp inclination $59^{\circ}$, palatal $50^{\circ}$ \\
2 & 10 & Buccal cusp inclination $64^{\circ}$, palatal $55^{\circ}$ \\
3 & 10 & Buccal cusp inclination $69^{\circ}$, palatal $60^{\circ}$ \\
4 & 10 & Control group (intact)
\end{tabular}

$\mathrm{NB}$, the buccal and palatal cusp inclinations are all in the $95 \%$ confidence interval.

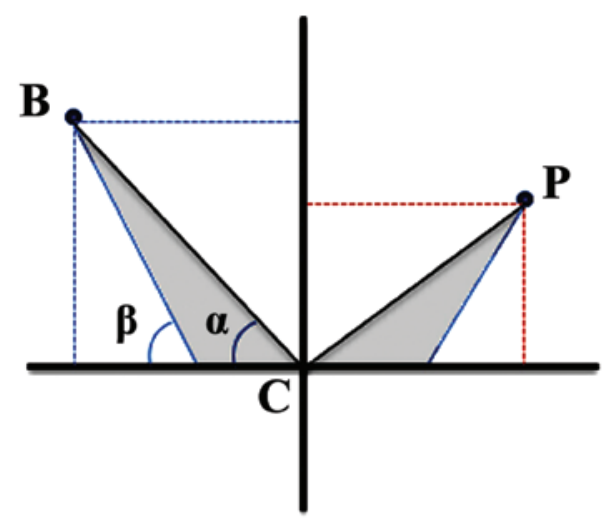

Figure 1. Planar mapping of extracted teeth: B, buccal cusp; P, palatalcusp; $\mathrm{C}$, central fossa; $\alpha$, baseline cusp inclination; $\beta$, adjusted cusp inclination with bilateral dentine removed (grey shade).

removed in order to keep more tooth tissues. The patents would fail to see the dentist timely when the symptom is not obvious. The clinical symptoms has a significant relationship with the crack size. Therefore, the cracks have already expanded when most patients want to receive the treatment. A tiny silicon carbide bur is used to prepare relatively larger crack for simulating clinical situation. All the samples were embedded in the type box, using silicone rubber to simulate periodontal membrane and self-setting resin to simulate alveolar bone.

Compression loading test. Prior to compression loading test, bite force test of Paris plaster molds of CTS by silicone rubber was performed, which revealed diverse biting contact sites, whereas the majority of bite contact sites were located in the middle and lower $1 / 3$ of cusp inclination. All groups underwent compression-loading test to determine the crack thresholds on an electronic universal material-testing machine. Briefly, the samples were mounted to the type boxes, with the teeth longitudinally vertical to the platform, and loader vertically against the contact site. The loader was advanced at a velocity of $2 \mathrm{~mm} / \mathrm{min}$ until the onset of fracture, with the fracture modes recorded and fracture risk scores calculated.

Statistical analysis. All analyses were performed using the SPSS version 16.0 (SPSS, Chicago, IL, USA). Data were expressed as mean $\pm \mathrm{SD}$. The rank sum test was used to compare the means of crack threshold among groups and $\mathrm{P}<0.05$ was considered statistically significant. 

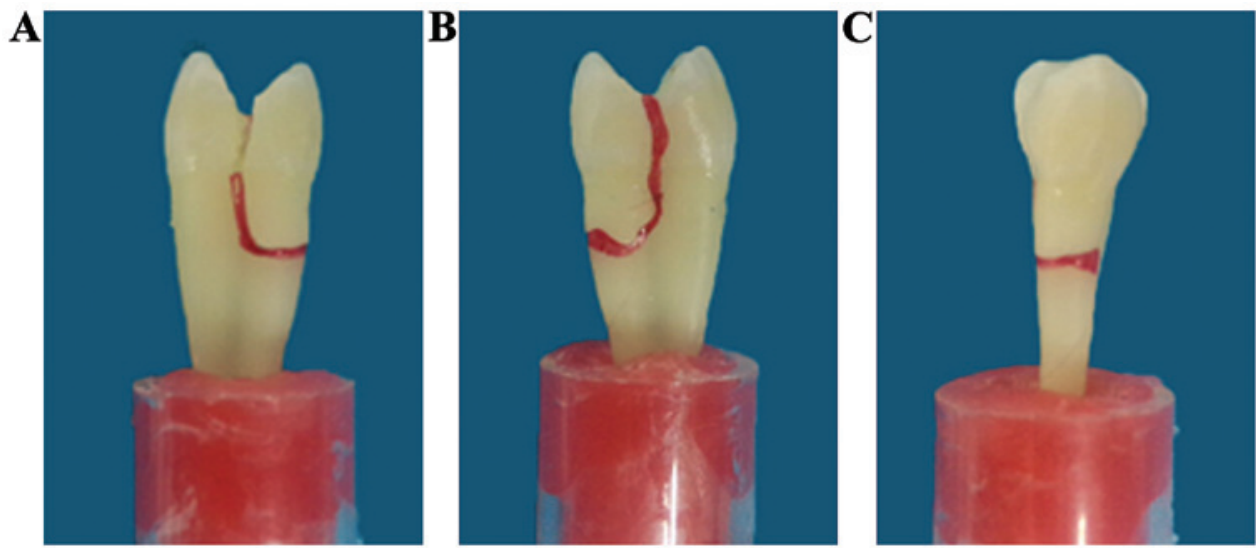

Figure 2. Fractures of cracked teeth in group I: (A) Crack extension down the pre-crack till $2 \mathrm{~mm}$ below cementoenamel junction (CEJ) and mesoradical 1/3 portion and transverse deflection. (B) Downward crack extension across the marginal ridge till $2 \mathrm{~mm}$ below CEJ and transverse deflection. (C) Fracture at supra- and mesoradical $1 / 3$ portion.
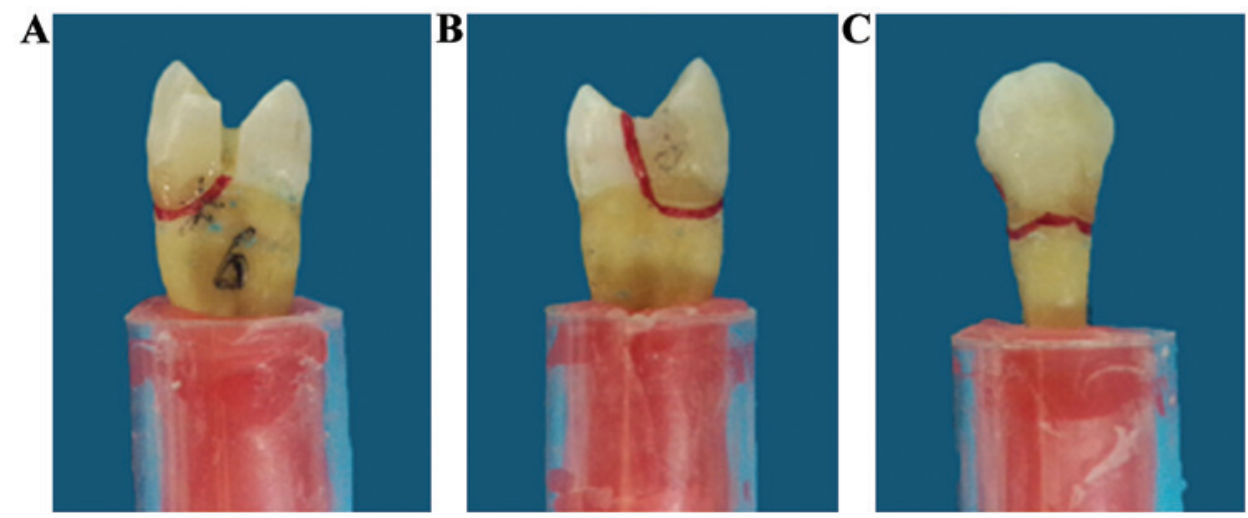

Figure 3. Fracture modes of cracked teeth in group II: (A) The buccolateral inclination of crack extension till cementoenamel junction (CEJ) and transverse deflection. (B) Downward crack extension across the marginal ridge till $2 \mathrm{~mm}$ below CEJ and mesoradical $1 / 3$ portion and transverse deflection. (C) Buccolateral fracture adjacent to the CEJ.
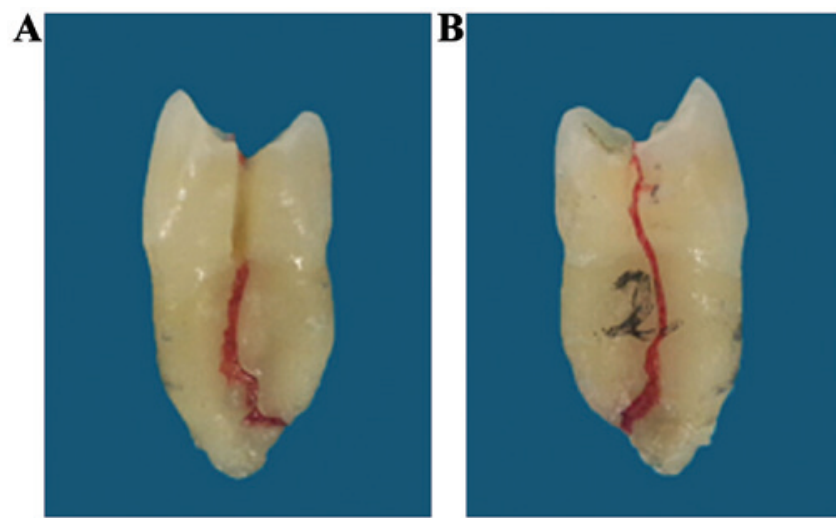

Figure 4. Fractures modes of cracked teeth in group III: (A) Longitudinal crack extension till the mesoradical portion and palatolateral deflection in downward echelons. (B) Longitudinal crack extension across the marginal ridge till mesoradical $1 / 3$ portion and meander by palatolateral inclination of deflection.

\section{Results}

Fracture modes. Samples in all groups underwent compression loading test, resulting in complete fracture (Figs. 2-5). The fracture modes and numbers in each group are depicted in Fig. 6.
With respect to fracture sites, groups I and II revealed a majority of fractures of corona radicis and a minority of coronal fractures, where groups III and IV exclusively exhibited coronal radical fractures and crown fracture, respectively; in terms of orientations and angles of fractures, oblique fractures were dominant in all groups, with a paucity of longitudinal fractures. With regard to fracture typology, CTS groups demonstrated complexity and variety in contrast to the control group.

In group I, the downward extension of cracks was either from the base of the pre-crack side or across the marginal ridge. The majority of cracks deflected palatolaterally from the site 1-2 $\mathrm{mm}$ below the CEJ, with a variety of deflect angles and a multitude of oblique fractures (Table II). Group II was comparable to group I in crack orientation, except for occasional crack extension from compression loading sites and a majority of palatolateral crown root fractures (Table III). Group III displayed a likewise crack extension till the CEJ, from where more complicated crack extensions arose: Either nearby deflection or radical progression and deflection at different angles and ultimately complete fractures resulted (Table IV). In addition, there were a majority of palatolaterally oblique fractures, with exclusive radical fractures in all samples. In the control group, oblique cracks generally extended palatolaterally downward from the compression loading sites at a variety 
Table II. Fracture modes of cracked teeth in group I.

Fracture modes

\begin{tabular}{|c|c|c|c|c|c|c|}
\hline Samples & Coronal & Radical & Palatal & Buccal & Oblique & Vertical \\
\hline 1 & & + & + & & + & \\
\hline 2 & & + & + & & + & \\
\hline 3 & & + & + & & & + \\
\hline 4 & & + & & + & + & \\
\hline 5 & & + & & + & + & \\
\hline 6 & & + & + & & + & \\
\hline 7 & + & & + & & + & \\
\hline 8 & & + & + & & + & \\
\hline 9 & & + & & + & + & \\
\hline 10 & + & & + & & + & \\
\hline Total & 2 & 8 & 7 & 3 & 9 & 1 \\
\hline
\end{tabular}
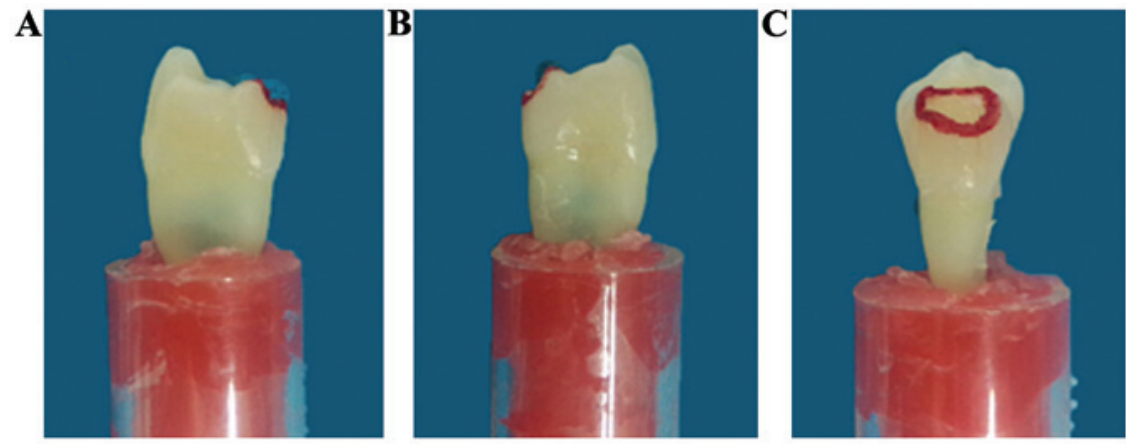

Figure 5. Fracture modes of cracked teeth in the control group: (A and B) Palatolateral inclination of crack extension from bite contact site. (C) Crack extension till mesocoronal $1 / 3$ portion with enamel fracture.

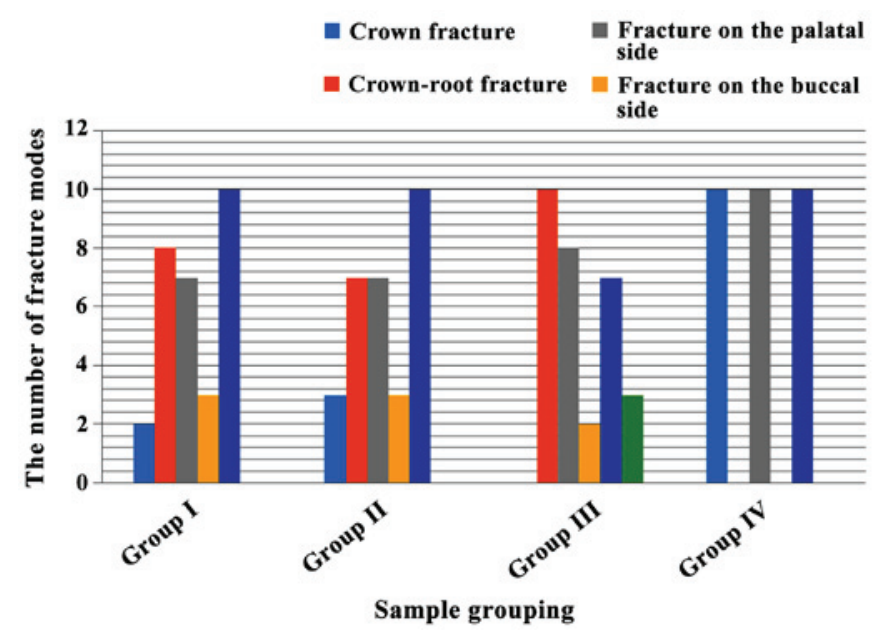

Figure 6. Fracture modes in each group.

of deflection angles, with enamel fracture mainly in mesocoronal or sub-coronal $1 / 3$ portions (Table V). All samples presented palatolateral coronal fractures (Fig. 6).

Estimation of fracture risk levels for cracked teeth. With reference to the de facto fracture modes of cracked teeth, the endodotists and dental restoration specialists formulated the fracture risk estimation scale (Table VI) of cracked teeth, which embodied findings from relevant studies (13) and provided practicable therapeutic options. Table VII illustrates the fracture risk scores in the four groups by the estimation scale. There were significant statistical differences in the fracture risk levels in group III and IV as compared with the other groups, respectively $(\mathrm{P}<0.05)$; the differences between groups I and II were insignificant $(\mathrm{P}>0.05)$. The fracture risk levels were similar in groups I and II; the fracture risk levels in group III were diverse and complicated, with higher scores for fracture risk levels and higher susceptibility of severe fractures. Group IV had the lowest scores for fracture risk levels and were the least susceptible for severe fractures.

\section{Discussion}

Dental anatomy could partially account for its susceptibility to fracture (19), with cusp inclination being one of the critical etiologies. Measurements of the cuspal inclinations on the radiograms of histological sections in each of the five posterior teeth samples revealed that cuspal inclination played a pivotal role in fracture potential and in the incidence of complete or incomplete cuspal fractures (20). Moreover, for the compromised teeth with CTS, steeper cuspal inclination 
Table III. Fracture modes of cracked teeth in group II.

Fracture modes

\begin{tabular}{|c|c|c|c|c|c|c|}
\hline Samples & Coronal & Radical & Palatolateral & Buccolateral & Oblique & Vertical \\
\hline 1 & + & & + & & + & \\
\hline 2 & & + & + & & + & \\
\hline 3 & & + & & + & + & \\
\hline 4 & & + & + & & + & \\
\hline 5 & & + & & + & + & \\
\hline 6 & & + & + & & + & \\
\hline 7 & & + & + & & + & \\
\hline 8 & & + & + & & + & \\
\hline 9 & + & & & + & + & \\
\hline 10 & & + & + & & + & \\
\hline Total & 3 & 7 & 7 & 3 & 10 & 0 \\
\hline
\end{tabular}

Table IV. Fracture modes of cracked teeth in group III.

\begin{tabular}{|c|c|c|c|c|c|c|}
\hline \multirow[b]{2}{*}{ Samples } & \multicolumn{6}{|c|}{ Fracture modes } \\
\hline & Coronal & Radical & Palatolateral & Buccolateral & Oblique & Vertical \\
\hline 1 & & + & & + & + & \\
\hline 2 & & + & + & & + & \\
\hline 3 & & + & + & & + & \\
\hline 4 & & + & + & & + & \\
\hline 5 & & + & + & & + & \\
\hline 6 & & + & + & & + & \\
\hline 7 & & + & + & & & + \\
\hline 8 & & + & & + & + & \\
\hline 9 & & + & + & & + & \\
\hline 10 & & + & + & & + & \\
\hline Total & 0 & 10 & 8 & 2 & 8 & 2 \\
\hline
\end{tabular}

frequently reportedly leads to an increment in tensile stress at the center groove and cervical region, predisposing to fracture formation (21). Three-dimensional finite element model (3-DFEM) has been applied in CTS study (22) and is well acknowledged for its advantages in evaluation of cuspal morphology with respect to mechanical analysis of stress distribution, whereas 3-DFEM is also noted for its limitations in terms of functional analysis, in which the majority of studies in CTS are restricted to loading stress analysis and studies of fracture modality is largely restrained (23). Given the status quo of 3-DFEM, our experiment was focused on the ex vivo teeth and simulated with endeavor the de facto endodontic environment so as to compensate for the drawbacks of 3-DFEM in CTS study.

Our experiment, with all the stress loading and fracture procedures visually recorded with high resolution, obtained the detailed data of the fracture modality of all the samples, which is unprecedented in CTS study. As for the sites of fracture onset, the cracks in CTS and control groups extended from pre-crack site and stress loading area, respectively. The difference in crack-onset sites is attributable to the distinctions in stress focus distribution. Based on the analysis of mechanics of materials, the stress-focused areas are the sites with defects of the materials per se or the stress-loaded, i.e. in the CTS group, the stress-focus was the pre-crack site, or rather, the area of dental defect, whereas in the control group, the contact site of the stress loader. The more complex and severe root fractures of the samples often occur in the CTS groups compared with control group. Cracked tooth always suffer splitting while the normal tooth have enamel defect clinically. The experimental results agree with the clinical observation. In the CTS groups, the sites of tooth fractures have the trend to move toward the root apex with the gradient increase of the cupsal inclinations, this may indicate that the steeper cupsal inclinations could lead to the more complex fracture pattern and more rugged splitting path, thus it plays an important role in the choice of treatment plan and prognosis.

Observation and analysis of the crack extension process revealed a multitude of oblique fractures with uneven routes characterized by deflection in arch or complex zig-zags, which 
Table V. Fracture modes of cracked teeth in group IV.

Fracture modes

\begin{tabular}{|c|c|c|c|c|c|c|}
\hline Samples & Coronal & Radical & Palatolateral & Buccolateral & Oblique & Vertical \\
\hline 1 & + & & + & & + & \\
\hline 2 & + & & + & & + & \\
\hline 3 & + & & + & & + & \\
\hline 4 & + & & + & & + & \\
\hline 5 & + & & + & & + & \\
\hline 6 & + & & + & & + & \\
\hline 7 & + & & + & & + & \\
\hline 8 & + & & + & & + & \\
\hline 9 & + & & + & & + & \\
\hline 10 & + & & + & & + & \\
\hline Total & 10 & 0 & 10 & 0 & 10 & 0 \\
\hline
\end{tabular}

Table VI. Scale for fracture risk levels of CTS and regimens.

\begin{tabular}{|c|c|c|}
\hline Levels & Criteria & Regimens \\
\hline I & Coronal fracture with intact dental pulp & $\begin{array}{l}\text { Occlusal adjustment; filling treatment; } \\
\text { indirect pulp capping; full crown capping }\end{array}$ \\
\hline II & Coronal fracture involving dental pulp & $\begin{array}{l}\text { Direct pulp capping; root canal treatment } \\
\text { and full crown capping }\end{array}$ \\
\hline III & Coronoradical fracture $\geq 2 \mathrm{~mm}$ above DEJ & Root canal treatment and full crown capping \\
\hline IV & $\begin{array}{l}\text { Coronoradical fracture at upper } 1 / 3 \text { portion of root, } \\
\text { the radical length } \geq 18 \mathrm{~mm}\end{array}$ & $\begin{array}{l}\text { Dental crown lengthening, root canal treatment } \\
\text { and full crown capping }\end{array}$ \\
\hline $\mathrm{V}$ & $\begin{array}{l}\text { Coronoradical fracture at upper } 1 / 3 \text { portion of root, } \\
\text { the radical length }<18 \mathrm{~mm}\end{array}$ & $\begin{array}{l}\text { Poor results, there is only a part of them } \\
\text { can be treated as level IV }\end{array}$ \\
\hline VI & Coronoradical fracture at middle and lower $2 / 3$ portion of root & Poor results, all of them should be extracted \\
\hline
\end{tabular}

Table VII. Scores for fracture risk levels of CTS.

\begin{tabular}{lllllll}
\hline Group & I & II & III & IV & V & VI \\
\hline I & 0 & 3 & 6 & 1 & 0 & 0 \\
II & 0 & 1 & 6 & 3 & 0 & 0 \\
III & 0 & 0 & 1 & 4 & 2 & 3 \\
IV & 4 & 6 & 0 & 0 & 0 & 0 \\
\hline
\end{tabular}

is correlated with the heterogeneity in dental composition and resilience involved. Intraenamel protein composites contribute to the diminished stress focus on the tip of enamel crack and enhanced resistance to crack and fracture (21). In addition, crack extension is typically countered with the increment in resistance of enamel, and the route of crack extension mainly rests on the extension interface with enamel and its microstructure (24), and there may be prism decussation adjacent to the EDJ (3), all of which contribute to the complexity of crack extension. All these findings of intradental microstructure may well account for the crack extension modality in our experiment, in which fracture is characteristically vertical or oblique, coupled with enamel exfoliation. Our results were consistent with the reports on dental defoliation and splitting that overload on the teeth would result in higher risk for fracture: longitudinal loading would extend the vertical crack downward to the dentin and penetrate to the dental base, causing splitting of a molar or premolar; chipping cracks can extend to the enameldentin interface, causing a partial fragmentation of a tooth; transverse cracks most notably in canines under lateral loading can directly penetrate into the subterranean dentin, rendering the tooth impaired (25).

In our experiment, enamel stripping is predominant in the control group, whereas the CTS group was dominated by crown-root fracture, which proportionally increases with the increment of cusp inclination, and the ultimate fracture site may exhibit a tendency of shifting towards the radical tip. We thus postulated that the cracked teeth are more prone to perpendicular fracture with the increment of cusp inclination to a certain angle, coupled with the enhanced complexity of fracture modes and routes. 
Numerous clinical therapeutic regimens are available regarding the CTS, such as occlusal adjustment, orthodontics and annulation, cusp and crown capping, depending on the clinical manifestations (26). In the case of fractures, however, cracked teeth should be evaluated for the value of conservation according to the severity. Accordingly, we formulated the fracture risk scale and provided relevant recommendations on the regimen options. Our experiment revealed that the control group had a high prevalence of coronal fractures, with fracture risks of I and II, for which unelaborated regimens suffice and the prognosis is good. The CTS group had a majority of crownroot fractures, which were categorized as high risks. The cusp inclinations in groups I and II were of relative minor angles, with the fracture risk of III in the majority of samples; group III had the greatest cusp inclinations, which had higher fracture risks and even risk of VI, i.e. complete vertical fracture till the root apex. The increment of cusp inclination to certain angles in CTS would increase the susceptibility to fracture of higher risks, which greatly hamper the therapeutic regimens and prognosis. In the case of severe fractures in CTS, particularly those beneath alveolar ridge crest, clinicians should meticulously evaluate the fracture sites and radical lengths and in certain cases, subsequent to root canal treatment, periodontal surgery is required prior to restorative therapy. Our experiment may provide recommendations to clinicians in examinations of CTS in addressing cusp inclinations. In cases of steeper cusp inclinations, adjustments for reduction should be made to obviate severe consequences as fracture. Additionally, our fracture risk scale may benefit clinicians in decision making as to rapid estimation of tooth conservation and provide patients with optimal regimen options.

This study has limitations in that our experiment utilized static stress algorithms to simulate the endodontic stress, which may create certain bias versus the de facto profiles. Moreover, our credibility and reliability are discounted due to the present sample size, which needs to be addressed in a future study.

\section{Acknowledgements}

In the present study, the pathogenesis and clinical research on cracked teeth of the innovation team was supported by the Health and Family Planning Commission of Xuzhou Municipal Government, Jiangsu Province, China (no. XWCX201609).

\section{References}

1. DeGusta D, Everett MA and Milton K: Natural selection on molar size in a wild population of howler monkeys (Alouatta palliata). Proc Biol Sci 270 (Suppl 1): S15-S17, 2003.

2. King SJ, Arrigo-Nelson SJ, Pochron ST, Semprebon GM, Godfrey LR, Wright PC and Jernvall J: Dental senescence in a long-lived primate links infant survival to rainfall. Proc Natl Acad Sci USA 102: 16579-16583, 2005.
3. Chai H, Lee JJ, Constantino PJ, Lucas PW and Lawn BR: Remarkable resilience of teeth. Proc Natl Acad Sci USA 106: 7289-7293, 2009.

4. Sui T, Lunt AJ, Baimpas N, Sandholzer MA, Li T, Zeng K, Landini $\mathrm{G}$ and Korsunsky AM: Understanding nature's residual strain engineering at the human dentine-enamel junction interface. Acta Biomater 32: 256-263, 2016.

5. Lee JJ, Constantino PJ, Lucas PW and Lawn BR: Fracture in teeth: A diagnostic for inferring bite force and tooth function. Biol Rev Camb Philos Soc 86: 959-974, 2011.

6. Geurtsen W, Schwarze T and Günay H: Diagnosis, therapy, and prevention of the cracked tooth syndrome. Quintessence Int 34: 409-417, 2003.

7. Lubisich EB, Hilton TJ and Ferracane J; Northwest Precedent: Cracked teeth: A review of the literature. J Esthet Restor Dent 22: 158-167, 2010.

8. Kim SY, Kim SH, Cho SB, Lee GO and Yang SE: Different treatment protocols for different pulpal and periapical diagnoses of 72 cracked teeth. J Endod 39: 449-452, 2013.

9. Lynch CD and McConnell RJ: The cracked tooth syndrome. J Can Dent Assoc 68: 470-475, 2002.

10. Davis R and Overton JD: Efficacy of bonded and nonbonded amalgam in the treatment of teeth with incomplete fractures. J Am Dent Assoc 131: 469-478, 2000.

11. Griffin JD Jr: Efficient, conservative treatment of symptomatic cracked teeth. Compend Contin Educ Dent 27: 93-102; quiz 103, $112,2006$.

12. Ratcliff S, Becker IM and Quinn L: Type and incidence of cracks in posterior teeth. J Prosthet Dent 86: 168-172, 2001.

13. Geurtsen W and García-Godoy F: Bonded restorations for the prevention and treatment of the cracked-tooth syndrome. Am J Dent 12: 266-270, 1999.

14. Chong BS: Bilateral cracked teeth: A case report. Int Endod J 22: 193-196, 1989.

15. Khers SC, Carpenter CW, Vetter JD and Staley RN: Anatomy of cusps of posterior teeth and their fracture potential. J Prosthet Dent 64: 139-147, 1990.

16. Qian Y, Zhou X and Yang J: Correlation between cuspal inclination and tooth cracked syndrome: a three-dimensional reconstruction measurement and finite element analysis. Dent Traumatol 29: 226-233, 2013.

17. Sadasiva K, Ramalingam S, Rajaram K and Meiyappan A: Cracked tooth syndrome: A report of three cases. J Pharm Bioallied Sci 7 (Suppl 2): S700-S703, 2015.

18. Banerji S, Mehta SB and Millar BJ: Cracked tooth syndrome. Part 2: Restorative options for the management of cracked tooth syndrome. Br Dent J 208: 503-514, 2010.

19. Bader JD, Shugars DA and Martin JA: Risk indicators for posterior tooth fracture. J Am Dent Assoc 135: 883-892, 2004.

20. Seo DG, Yi YA, Shin SJ and Park JW: Analysis of factors associated with cracked teeth. J Endod 38: 288-292, 2012.

21. Barani A, Chai H, Lawn BR and Bush MB: Mechanics analysis of molar tooth splitting. Acta Biomater 15: 237-243, 2015.

22. Imanishi A, Nakamura $T$, Ohyama $T$ and Nakamura $T$ : 3-D Finite element analysis of all-ceramic posterior crowns. J Oral Rehabil 30: 818-822, 2003.

23. Ji B and Gao H: A study of fracture mechanisms in biological nano-composites via the virtual internal bond model. Mater Sci Eng A 366: 96-103, 2004.

24. Padmanabhan SK, Balakrishnan A, Chu MC, Kim TN and Cho SJ: Micro-indentation fracture behavior of human enamel. Dent Mater 26: 100-104, 2010.

25. Chai H, Lee JJ and Lawn BR: On the chipping and splitting of teeth. J Mech Behav Biomed Mater 4: 315-321, 2011.

26. Turp JC and Gobetti JP: The cracked tooth syndrome: An elusive diagnosis. J Am Dent Assoc 127: 1502-1507, 1996. 